\title{
ITERATION OF HOLOMORPHIC MAPS OF THE UNIT BALL INTO ITSELF
}

\author{
YOSHIHISA KUBOTA \\ Dedicated to Professor Mitsuru Ozawa \\ on the occasion of his 60 th birthday
}

\begin{abstract}
Let $\Omega$ be a plane disc and let $f$ be a holomorphic map of $\Omega$ into itself. It is known that the iterates $f_{n}$ of $f$ converge to a constant $\zeta \in \bar{\Omega}$ as $n \rightarrow \infty$ unless $f$ is a conformal map of $\Omega$ onto itself. In the present paper it is shown that a more complicated statement of this kind is true in the unit ball of $\mathbf{C}^{N}$.
\end{abstract}

1. Let $\Omega$ be a plane disc and let $f$ be a holomorphic map of $\Omega$ into itself. It is known that the iterates $f_{n}$ of $f$ converge to a constant $\zeta \in \bar{\Omega}$ as $n \rightarrow \infty$ unless $f$ is a conformal map of $\Omega$ onto itself [3, pp. 131-134]. In the present paper we study the action of the iterates of holomorphic maps of the unit ball into itself.

Let $B_{N}$ be the unit ball of $C^{N}$ and let $F$ be a holomorphic map of $B_{N}$ into itself. The iterates $F_{n}$ of $F$ are defined by

$$
F_{0}(z)=z, \quad F_{n+1}(z)=F\left(F_{n}(z)\right) \quad(n=0,1, \ldots) .
$$

Let $r$ be a positive integer with $1 \leqslant r \leqslant N$. Corresponding to the orthogonal direct sum decomposition $\mathbf{C}^{N}=\mathbf{C}^{r} \oplus \mathbf{C}^{N-r}$, each $z \in \mathbf{C}^{N}$ decomposes into $z=z^{\prime}+z^{\prime \prime}$, where $z^{\prime} \in \mathbf{C}^{r}, z^{\prime \prime} \in \mathbf{C}^{N-r}$. Accordingly, for a map $\Phi=\left(\phi_{1}, \ldots, \phi_{N}\right)$ from $B_{N}$ into $\mathrm{C}^{N}$, we write $\Phi=\Phi^{\prime}+\Phi^{\prime \prime}$, where $\Phi^{\prime}=\left(\phi_{1}, \ldots, \phi_{r}\right)$ and $\Phi^{\prime \prime}=\left(\phi_{r+1}, \ldots, \phi_{N}\right)$.

THEOREM. Let $F$ be a holomorphic map of $B_{N}$ into itself. If $F$ is not an automorphism of $B_{N}$, then either

(i) the iterates $F_{n}$ of $F$ converge to a constant map as $n \rightarrow \infty$, uniformly on every compact subset of $B_{N}$, or

(ii) there exist a subsequence $\left\{F_{n_{v}}\right\}$ of $\left\{F_{n}\right\}$, an automorphism $T$ of $B_{N}$ and a positive integer $r$ with $1 \leqslant r<N$, such that $T \circ F_{n_{v}} \circ T^{-1}$ converge as $v \rightarrow \infty$, uniformly on every compact subset of $B_{N}$, to a holomorphic map $\Phi$ of $B_{N}$ into itself having the following properties: (a) $\Phi^{\prime}\left(z^{\prime}\right)=z^{\prime}$ for $z^{\prime} \in B_{r}$ and (b) $\Phi^{\prime \prime}(z)=0^{\prime \prime}$ for $z \in B_{N}$.

In case (i), if $\zeta=\lim _{n \rightarrow \infty} F_{n}(z), z \in B_{N}$, is a point in $B_{N}$, then $\zeta$ is the fixed point of F.

In case (ii) the restriction $\tilde{F}^{\prime}\left(z^{\prime}\right)$ of $\tilde{F}=T \circ F \circ T^{-1}$ to $B_{r}$ is an automorphism of $B_{r}$.

Received by the editors June 23, 1982 and, in revised form, September 9, 1982.

1980 Mathematics Subject Classification. Primary 32H99.

Key words and phrases. Iteration, holomorphic map, unit ball.

(C) 1983 American Mathematical Society 0002-9939/82/0000-0946/\$01.75 
There is a simple example which belongs to case (ii). We define the map $F=\left(f_{1}, f_{2}\right)$ by

$$
f_{1}(z)=-z_{1}+a z_{2}^{2}, \quad f_{2}(z)=b z_{2}, \quad z=\left(z_{1}, z_{2}\right),
$$

where $2|a|+|a|^{2}+|b|^{2}<1$. Then $F$ is a holomorphic map of $B_{2}$ into itself and $F_{2 k}$ converge as $k \rightarrow \infty$ to the map

$$
\Phi(z)=\left(z_{1}-\frac{a}{1+b^{2}} z_{2}^{2}, 0\right)
$$

and $F_{2 k+1}$ converge as $k \rightarrow \infty$ to the map

$$
\psi(z)=\left(-z_{1}+\frac{a}{1+b^{2}} z_{2}^{2}, 0\right) .
$$

Finally we note that there is a holomorphic map $F$ of the unit polydisc $U^{N}$ in $\mathbf{C}^{N}$ into itself such that the iterates $F_{n}$ converge to a nonconstant map from $U^{N}$ into $\partial U^{N}$. A simple example is

$$
F(z)=\left(\frac{2}{3} z_{1}+\frac{1}{3}, z_{2}, \ldots, z_{N}\right), \quad z=\left(z_{1}, \ldots, z_{N}\right) .
$$

2. We begin with an elementary lemma.

LEMMA. Let $\Psi$ be a holomorphic map from $B_{N}$ into $\bar{B}_{N}$. If $\Psi$ is not constant, then $\Psi\left(B_{N}\right) \subset B_{N}$.

Proof. Suppose that $\Psi=\left(\psi_{1}, \ldots, \psi_{N}\right)$ maps one point in $B_{N}$ to a point $w$ in the boundary of $B_{N}$. We may assume that $w=(1,0, \ldots, 0)$. Then the maximum principle shows that $\psi_{1}(z) \equiv 1$, and so that $\psi_{j}(z) \equiv 0, j=2, \ldots, N$. Thus $\Psi$ must be constant.

Let $\Psi$ be a holomorphic map from $B_{N}$ into $\mathrm{C}^{N}$. We denote by $A_{\Psi}(z)$ the matrix $\left(a_{i j}\right)$,

$$
a_{i j}=\frac{\partial \psi_{i}}{\partial z_{j}}(z) \quad(1 \leqslant i \leqslant N, 1 \leqslant j \leqslant N)
$$

where $\Psi=\left(\psi_{1}, \ldots, \psi_{N}\right)$ and $z=\left(z_{1}, \ldots, z_{N}\right)$.

3. We now turn to the proof of the theorem. Let $F$ be a holomorphic map of $B_{N}$ into itself and let $F_{n}$ be its $n$th iterate. Then the sequence $\left\{F_{n}\right\}$ is normal in $B_{N}$.

Case 1. Every convergent subsequence of $\left\{F_{n}\right\}$ converges to a constant map uniformly on every compact subset of $B_{N}$.

Case 1.1. There exists a subsequence $\left\{F_{n_{v}}\right\}$ converging to a constant map which maps $B_{N}$ into a point $\zeta$ in $B_{N}$.

We have

$$
F(\zeta)=\lim _{v \rightarrow \infty} F\left(F_{n_{v}}(z)\right)=\lim _{v \rightarrow \infty} F_{n_{v}}(F(z))=\zeta
$$

and, hence, for any convergent subsequence $\left\{F_{m_{v}}\right\}$ we have

$$
\lim _{v \rightarrow \infty} F_{m_{v}}(z)=\lim _{v \rightarrow \infty} F_{m_{v}}(\zeta)=\zeta \quad\left(z \in B_{N}\right) .
$$

Thus, in this case, $\left\{F_{n}\right\}$ converges to the constant $\zeta$ and $\zeta$ is the fixed point of $F$. 
Case 1.2. Every convergent subsequence of $\left\{F_{n}\right\}$ converges to a constant map which maps $B_{N}$ into a point in the boundary of $B_{N}$.

In this case we have $\left\|F_{n}\left(z^{*}\right)\right\| \rightarrow 1$ as $n \rightarrow \infty$, where $z^{*}$ is a point in $B_{N}$. Hence there exists a subsequence $\left\{F_{n_{v}}\right\}$ such that

$$
\left\|F_{n_{v}}\left(z^{*}\right)\right\|<\left\|F_{n_{v}+1}\left(z^{*}\right)\right\| \quad(v=1,2, \ldots) .
$$

We may assume that $\left\{F_{n_{t}}(z)\right\}, z \in B_{N}$, converges to the point $e_{1}=(1,0, \ldots, 0)$. Put $a_{v}=F_{n_{v}}\left(z^{*}\right)(v=1,2, \ldots)$. Then

$$
\begin{gathered}
\left\|a_{v}\right\|<\left\|F\left(a_{v}\right)\right\| \quad(v=1,2, \ldots), \\
\lim _{v \rightarrow \infty} a_{v}=e_{1}, \quad \lim _{v \rightarrow \infty} F\left(a_{v}\right)=\lim _{v \rightarrow \infty} F_{n_{v}}\left(F\left(z^{*}\right)\right)=e_{1} .
\end{gathered}
$$

Hence, letting $v \rightarrow \infty$ in the inequality

$$
\frac{\left|1-\left\langle F\left(a_{v}\right), F(z)\right\rangle\right|^{2}}{1-\|F(z)\|^{2}} \leqslant \frac{1-\left\|F\left(a_{v}\right)\right\|^{2}}{1-\left\|a_{v}\right\|^{2}} \frac{\left|1-\left\langle a_{v}, z\right\rangle\right|^{2}}{1-\|z\|^{2}} \quad\left(z \in B_{N}\right)
$$

$[2$, p.163] we have

$$
\frac{\left|1-f_{1}(z)\right|^{2}}{1-\|F(z)\|^{2}} \leqslant \frac{\left|1-z_{1}\right|^{2}}{1-\|z\|^{2}} \quad\left(z \in B_{N}\right)
$$

where $F=\left(f_{1}, \ldots, f_{N}\right)$ and $z=\left(z_{1}, \ldots, z_{N}\right)$. Then writing $F_{n}=\left(f_{1}^{(n)}, \ldots, f_{N}^{(n)}\right)$ we obtain

$$
\frac{\left|1-f_{1}^{(n)}(z)\right|^{2}}{1-\left\|F_{n}(z)\right\|^{2}} \leqslant \frac{\left|1-z_{1}\right|^{2}}{1-\|z\|^{2}} \quad\left(z \in B_{N}\right) \text {. }
$$

Since $\left\|F_{n}(z)\right\| \rightarrow 1$ as $n \rightarrow \infty$, it follows from this inequality that $f_{1}^{(n)}(z) \rightarrow 1$ as $n \rightarrow \infty$. This shows that $F_{n}(z) \rightarrow e_{1}$ as $n \rightarrow \infty$.

Case 2. There exists a subsequence $\left\{F_{n_{v}}\right\}$ which converges to a nonconstant map $\Psi$, uniformly on every compact subset of $B_{N}$.

There is a point $z^{*}$ in $B_{N}$ with rank $A_{\Psi}\left(z^{*}\right)>0$. We may assume that

$$
\operatorname{rank} A_{\Psi}\left(z^{*}\right)=\sup _{\Phi \in \mathcal{L}} \sup _{z \in B_{N}} \operatorname{rank} A_{\Phi}(z) \equiv r \leqslant N,
$$

where $\mathcal{L}$ is the family of all limit maps of convergent subsequences of $\left\{F_{n}\right\}$. Further we may assume that $\left\{F_{n_{v+1}-n_{v}}\right\}$ also converges. Let $\Phi$ be its limit map. Since $\Psi\left(B_{N}\right) \subset B_{N}$ (it follows from the lemma), we have

$$
\Phi(\Psi(z))=\lim _{v \rightarrow \infty} F_{n_{v+1}-n_{v}}\left(F_{n_{v}}(z)\right)=\Psi(z) \quad\left(z \in B_{N}\right) .
$$

Putting $w^{*}=\Psi\left(z^{*}\right)$ we have $\Phi\left(w^{*}\right)=w^{*}$. Regarding $A_{\Psi}$ and $A_{\Phi}$ as linear operators, rather than matrices, the relation $\Phi \circ \Psi=\Psi$ shows that $A_{\Phi}\left(w^{*}\right) \circ A_{\Psi}\left(z^{*}\right)=A_{\Psi}\left(z^{*}\right)$. This shows that the restriction of $A_{\Phi}\left(w^{*}\right)$ to the $r$-dimensional space which is the range of $A_{\Psi}\left(z^{*}\right)$ is the identity. Hence, by the definition of $r$, it follows that $\operatorname{rank} A_{\Phi}\left(w^{*}\right)=r$. 
Now we may assume that $\Phi(0)=0$ and $A_{\Phi}(0)=\left(\begin{array}{cc}I & X \\ Y & Z\end{array}\right)$, rank $A_{\Phi}(0)=r$, by considering the map $T \circ F \circ T^{-1}$ in place of $F$, where $I$ is the identity matrix of order $r$ and $T$ is a suitable automorphism of $B_{N}$.

We shall show that $\Phi$ has properties (a) and (b). Since $\Phi^{\prime}\left(z^{\prime}\right)$ is a holomorphic map of $B_{r}$ into itself with $\Phi^{\prime}\left(0^{\prime}\right)=0^{\prime}$ and since $A_{\Phi^{\prime}}\left(0^{\prime}\right)=I$, it follows by Cartan's uniqueness theorem that

$$
\Phi^{\prime}\left(z^{\prime}\right)=z^{\prime} \quad\left(z^{\prime} \in B_{r}\right) .
$$

Further, from the inequality

$$
\left\|z^{\prime}\right\|^{2}+\left\|\Phi^{\prime \prime}\left(z^{\prime}\right)\right\|^{2}=\left\|\Phi\left(z^{\prime}\right)\right\|^{2}<1 \quad\left(z^{\prime} \in B_{r}\right)
$$

we have that $\left\|\Phi^{\prime \prime}\left(z^{\prime}\right)\right\| \rightarrow 0$ as $\left\|z^{\prime}\right\| \rightarrow 1$ (where \|\| denotes the euclidean norm), and so

$$
\Phi^{\prime \prime}\left(z^{\prime}\right)=0^{\prime \prime} \quad\left(z^{\prime} \in B_{r}\right) .
$$

On the other hand, since rank $A_{\Phi}(z)=r$ in a neighborhood $U$ of 0 , there exist functions $H_{j}\left(z^{\prime}\right), j=r+1, \ldots, N$, such that $H_{j}\left(z^{\prime}\right)$ are holomorphic in a neighborhood $U^{\prime}$ of $0^{\prime}$ and

$$
\Phi^{\prime \prime}(z)=\left(H_{r+1}\left(\Phi^{\prime}(z)\right), \ldots, H_{N}\left(\Phi^{\prime}(z)\right)\right) \quad(z \in U) .
$$

(For instance, this follows from Theorem 5 in Chapter I, §B of [1].) From (1), (2) and (3) we see that

$$
\begin{aligned}
\left(H_{r+1}\left(z^{\prime}\right), \ldots, H_{N}\left(z^{\prime}\right)\right) & =\left(H_{r+1}\left(\Phi^{\prime}\left(z^{\prime}\right)\right), \ldots, H_{N}\left(\Phi^{\prime}\left(z^{\prime}\right)\right)\right) \\
& =\Phi^{\prime \prime}\left(z^{\prime}\right)=0^{\prime \prime} \quad\left(z^{\prime} \in U^{\prime}\right) .
\end{aligned}
$$

Hence $H_{j} \equiv 0, j=r+1, \ldots, N$, so that we obtain

$$
\Phi^{\prime \prime}(z)=0^{\prime \prime} \quad\left(z \in B_{N}\right) .
$$

Thus we have proved that there exist a subsequence $\left\{F_{n_{t}}\right\}$ (we consider $n_{v+1}-n_{v}$ as $n_{v}$ ), and an automorphism $T$ of $B_{N}$ such that $T \circ F_{n_{v}} \circ T^{-1}$ converge as $v \rightarrow \infty$ to a map $\Phi$ with properties (a) and (b).

Next we shall show that $\tilde{F}^{\prime}\left(z^{\prime}\right)$ is an automorphism of $B_{r}$, where $\tilde{F}=T \circ F \circ T^{-1}$ $=\tilde{F}^{\prime}+\tilde{F}^{\prime \prime}$. We assume that $\left\{F_{n_{v}-1}\right\}$ converges, without loss of generality. Putting $G=\lim _{v \rightarrow \infty} F_{n_{v}-1}$ and $\tilde{G}=T \circ G \circ T^{-1}=\tilde{G}^{\prime}+\tilde{G}^{\prime \prime}$ we have

$$
\tilde{G} \circ \tilde{F}=\lim _{v \rightarrow \infty} T \circ F_{n_{v}} \circ T^{-1}=\Phi .
$$

From this and the lemma it follows that $\tilde{G}\left(B_{N}\right) \subset B_{N}$. Hence we also obtain

$$
\tilde{F} \circ \tilde{G}=\Phi \text {. }
$$

Then we see from (1), (4), (5) and (6) that

$$
\tilde{G}^{\prime}\left(\tilde{F}\left(z^{\prime}\right)\right)=\tilde{F}^{\prime}\left(\tilde{G}\left(z^{\prime}\right)\right)=z^{\prime} \quad\left(z^{\prime} \in B_{r}\right)
$$

and

$$
\tilde{G}^{\prime \prime}(\tilde{F}(z))=\tilde{F}^{\prime \prime}(\tilde{G}(z))=0^{\prime \prime} \quad\left(z \in B_{N}\right)
$$


From (8) we obtain that $\tilde{F}^{\prime \prime}\left(z^{\prime}\right)=0^{\prime \prime}, z^{\prime} \in B_{r}$. Indeed, putting $w=\tilde{F}\left(z^{\prime}\right)$ we have

$$
\tilde{F}^{\prime \prime}\left(z^{\prime}\right)=\tilde{F}^{\prime \prime}\left(\Phi^{\prime}\left(z^{\prime}\right)\right)=\tilde{F}^{\prime \prime}(\tilde{G}(w))=0^{\prime \prime} .
$$

Similarly we obtain that $\tilde{G}^{\prime \prime}\left(z^{\prime}\right)=0^{\prime \prime}, \mathrm{z}^{\prime} \in B_{r}$. Therefore from (7) we have

$$
\tilde{G}^{\prime}\left(\tilde{F}^{\prime}\left(z^{\prime}\right)\right)=\tilde{F}^{\prime}\left(\tilde{G}^{\prime}\left(z^{\prime}\right)\right)=z^{\prime} \quad\left(z^{\prime} \in B_{r}\right) .
$$

This shows that the map $\tilde{F}^{\prime}\left(z^{\prime}\right)$ is an automorphism of $B_{r}$.

If $r=N$, then $F$ is an automorphism of $B_{N}$. Thus the proof of the theorem is complete.

\section{REFERENCES}

1. R. Gunning and H. Rossi, Analytic functions of several complex variables, Prentice-Hall, Englewood Cliffs, N.J., 1965.

2. W. Rudin, Function theory in the unit ball of $C^{n}$, Die Grundlehren der Math. Wissenschaften, Bd. 241, Springer-Verlag, Berlin, 1980.

3. G. Valiron, Fonctions analytiques, Presses Universitaires de France, Paris, 1954.

Department of Mathematics, Tokyo Gakugei University, Koganei-Shi, Tokyo 184, Japan 